\title{
Pupillary responses in a pitch-discrimination task
}

\author{
DANIEL KAHNEMAN 1, CENTER FOR COGNITIVE STUDIES, HARVARD UNIVERSITY \\ JACKSON BEATTY, HUMAN PERFORMANCE CENTER, UNIVERSITY OF MICHIGAN
}

Pupils were measured while observers made pitch judgments under the method of constant stimuli. A substantial dilation occurs immediately after the presentation of the comparison tone, and the size of this response is closely correlated to the difficulty of the discrimination. Baseline changes occur within each block of trials, but have little effect on the magnitude of dilations. Responses to redundant stimuli, including the standard itself, decrease during the experimental session. The results support the validity of pupillary measurements as an index of processing load.

Hess and Polt (1964) suggested that the pupillary response provides a sensitive peripheral indicator of the effort exerted during problem solving. Additional work has confirmed that suggestion and has further indicated that changes of pupil size may follow rapid variations in attention during a single task. Thus, in a short-term memory task, a pupillary dilation occurs as $\mathrm{S}$ attends to, and presumably rehearses, a string of digits or words (Kahneman \& Beatty, 1966). When a person performs a transformation on a string of digits, the dilation is substantially larger than when he merely repeats the string. The peak is attained during the reporting phase, which is also the time when the transformed string is produced and the processing load on the subject is maximal. Transient pupillary responses also reflect the different kinds of effort involved in thinking about concrete or abstract words (Paivio \& Simpson, 1966) or in recalling a familiar association (Beatty \& Kahneman, 1966). In the latter case, a very steep dilation occurs immediately after the presentation of the cue to report, and further work has shown that the pupillary response to the effort of recalling an item diminishes as the item is overlearned.

Although the foregoing results suggest a correlation between pupillary diameter and such constructs as arousal, alertness, or attention, it is apparent that the correlation is in need of additional specification. A person is certainly in a relatively heightened state of alertness when he is watching for a faint signal, intent on a melody, attempting to recall his wedding anniversary, or doing arithmetic in his head. Though all these states are presumably similar in some respects, they appear to differ in others. There is a consequent need to make more specific the relations between physiological indicators and various kinds of attention. An important distinction has lately been drawn between attention directed outward, as in listening to a melody, or inward as in solving a problem (Lacey, Kagan, Lacey, \& Moss, 1963; Obrist, 1963). Distinct physiological patterns of autonomic activation are correlated with those two states. The evidence available to date suggests that pupillary dilations occur whenever attention is directed to the active processing of information, but evidence is lacking concerning pupillary behavior when attention is directed to the passive acceptance of stimulation.

The present research extends the study of the correlation between pupillary diameter and processing load to a processing activity that is largely nonverbal in nature. Another objective was to compare the pupillary changes during a period of alert expectation with the changes that accompany the making of a decision after the information has been received. The study was also designed to show how pupillary responses change with practice in a discrimination task, and to separate significant changes from possible habituation artifacts.

\section{Method}

Ss were 10 paid student volunteers, 5 of them male. Each $S$ had 55 trials on a pitch discrimination task. A trial consisted of a sequence of four tones, each $250 \mathrm{msec}$. in duration, presented from a tape recorder through calibrated earphones at a sound pressure of $83 \mathrm{~dB}$. The first tone $(650 \mathrm{cps})$ was a ready signal; it was followed by a standard tone of $850 \mathrm{cps}$ after an interval that could vary between 2 and 6 sec.; a comparison tone was presented $4 \mathrm{sec}$. later, at a frequency that varied between 820 and $880 \mathrm{cps}$ in steps of $6 \mathrm{cps}$. Finally, after an additional delay of 4 sec., a $650 \mathrm{cps}$ tone indicated that $S$ was to report whether the comparison tone was higher or lower in pitch than the standard. $S$ was immediately told whether this judgment was correct or incorrect. In the case of the $850 \mathrm{cps}$ tone, he was always told that his answer had been correct. The ready signal for the subsequent trial was heard after an interval of 6-10 sec. following the instruction to respond. $S$ was instructed to refrain from blinking except during this period. The procedure was broken into five blocks of 11 trials, each including the different frequencies of the comparison tone. The order of frequencies and the durations of the two variable delays were randomly determined within each block, and the order of presentation of trial blocks was different for successive Ss. Each block was followed by a brief pause.

Photographs of S's left pupil and iris were taken on high-speed infrared film with a Grass C4-D camera, at a rate of $1 / \mathrm{sec}$. Exposures were made with a General Radio Strobolume filtered for infrared. The strobe was controlled by the tape that presented the auditory stimuli, thereby insuring synchrony between 


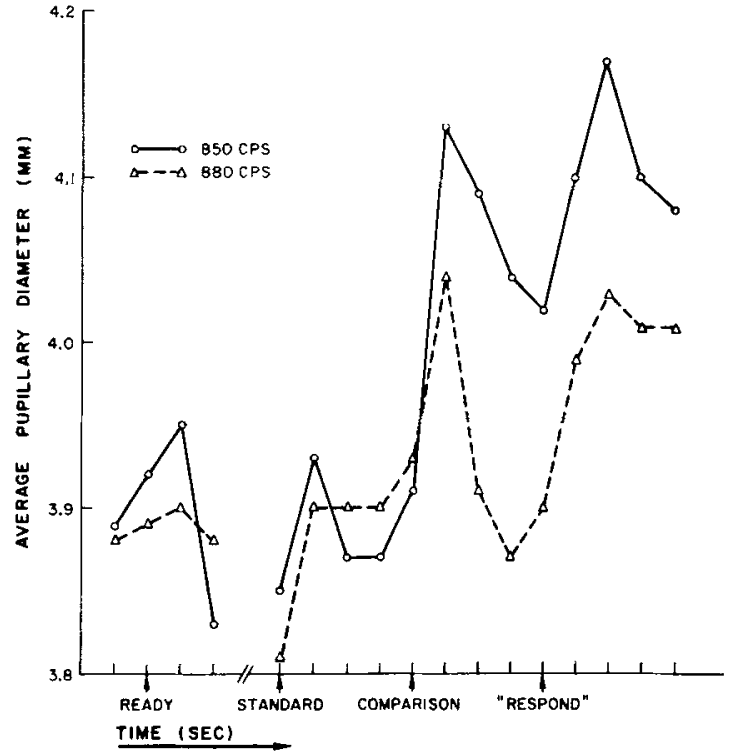

Fig. 1. Average pupillary diameter for $10 \mathrm{Ss}$ (5 trials per $\mathrm{S}$ ) for two discrimination tagks. The frequency of the comparison tone is the parameter. Standard tone frequency is always $850 \mathrm{cps}$.

the sequence of presentations and the sequence of pictures. S's right eye was occluded during the procedure. Additional lllumination to his left eye was provided by two fluorescent $15 \mathrm{w}$ bulbs placed $1 \mathrm{ft}$. away, in his peripheral field of view.

\section{Results}

Figure 1 presents the sequence of pupillary responses, averaged over trials and individuals, for trials on which the frequency of the comparison tone is $850 \mathrm{cps}$ (identical to the standard) and $880 \mathrm{cps}$ (the easlest discrimination).

The ready signal is followed by an immediate and transitory dilation. The occurrence of this dilation is reliable $(t=2.98, d f=9, p<.05)$, but its magnitude is small. Pupil diameter has returned to baseline 2 sec. after the presentation of the ready signal, and there is little further change until the presentation of the comparison tone. Thus the expectation of the standard tone appears to have no consistent effect on the pupil. It may be seen in Fig. 1 that the anticipation of the comparison tone, which always followed the standard after an interval of 4 sec., is associated with a slight dilation, which is statistically quite reliable $(t=4.35, p<.01)$.

The major difference between the method of constant stimuli and the method of single stimuli, is that a standard tone is presented in one case and not in the other (Stevens, 1958). However, S need pay only little attention to the standard tone in the method of constant stimuli, since he soon discovers that this tone is repeated on every trial (Wickelgren, 1966). The critical information is presented by the comparison tone, which accordingly elicits a larger dilation. Figure 1 shows a major difference between the responses to a standard and a comparison tone which are identical in frequency $(t=8.46, p<.001)$.

It may be further noted in Fig. 1 that the dilation increases with the difficulty of the discrimination: the $850 \mathrm{cps}$ tone elicits a much larger response than does the $880 \mathrm{cps}$ tone, and the difference is sustained untll the completion of the trial.

Additional evidence to this point is presented in Fig. 2, which shows percent errors and puplllary dilations to the comparison tone as functions of tonal frequency. The quadratic trend relating the magnitude of the pupillary response to the frequency of the comparison tone is highly significant $(F=25.78, \mathrm{df}=$ $1 / 90, p<.001$ ). In fact, the pupillary variable appears to be almost as good an index of the difficulty of discrimination as is the rate of errors.

The close relation between difficulty of discrimination and the magnitude of the pupillary response confirms the major hypothesis of this study, and extends the validity of pupillary diameter as an index of processing load to cases where the verbal content of the process is presumably minimal. In addition, the temporal course of the pupillary response corresponds to the subjective reports of the Ss that a decision was attained within a second or two after the presentation of the comparison tone. The secondary

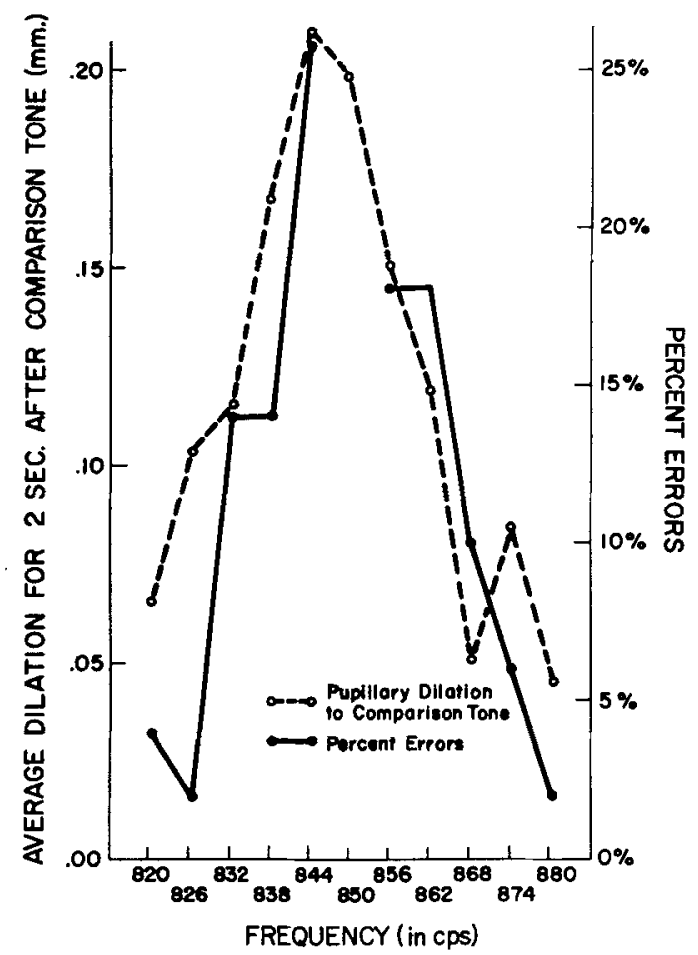

Fig. 2. Broken line: average dilation during 2 sec. following comparison tone as a function of frequency of the comparison tone. Solid line: percent errors. 
peak of the tracing corresponds to the effort involved in organizing the verbal response and emitting it in correct timing.

It may be noted in Fig. 1 that the secondary peak elicited by the instruction to respond is of similar magnitude for the two responses. There is in fact no association between the magnitude of this dilation and frequency $(F=1.81, d f=10 / 90, N S)$. On the other hand, the curves for 850 and $880 \mathrm{cps}$ remain essentially parallel. This result is also characteristic: the tracings for all frequencies show a baseline shift which is closely related to the difficulty of the discrimination and begins to fade only during the concluding 2 sec. of the trial. This baseline shift may be interpreted in at least three ways: it may represent the occurrence of continued hesitation and delijeration following the more difficult discriminations; it could also be due to an emotional component of anxious anticipation of E's reaction. Finally, it may be noted that the conditions during this period are paradigmatic for the occurrence of the expectancy wave in evoked potentials. This wave has been described by Walter (1964) as occurring when S alertly anticipates an imperative signal to produce a response.

In these analyses, all pupillary responses to a particular frequency were included, regardless of whether S's judgment was correct or incorrect. Essentially identical results are obtained when only correct responses are considered. In fact, there are no significant differences between responses on correct and incorrect trials, with one interesting exception: the announcement by the experimenter that S's answer was correct is typically followed by a pupillary constriction, while the announcement that the response was wrong elicits a dilation; the difference between these responses is highly significant $(t=3.92, p<.01)$. Again, the dilation following an incorrect answer could be an emotional reaction, although it may be interpreted more parsimoniously as representing an effort by the $S$ to recall the sequence of tones and to learn from the experience.

We now turn to an examination of progressive pupillary changes in the course of the experiment. The most striking of these changes is a pronounced pupillary constriction that occurs during every block of trials. Figure 3 presents the average pupil size of Ss at the instant of presentation of the ready signal for each of the 55 trials of the experiment. The magnitude of the dilation to each ready signal is also shown. It is apparent that the within-block constriction represents a continuous change of baseline; the more transient changes, which have been illustrated in Fig. 1, are evidently superimposed on the changing baseline. We have observed similar constrictions under other conditions in which $S$ is continuously exposed to a high level of illumination under instructions to refrain from blinking. A brief rest period during which $\mathrm{S}$ is free to blink and looks away from

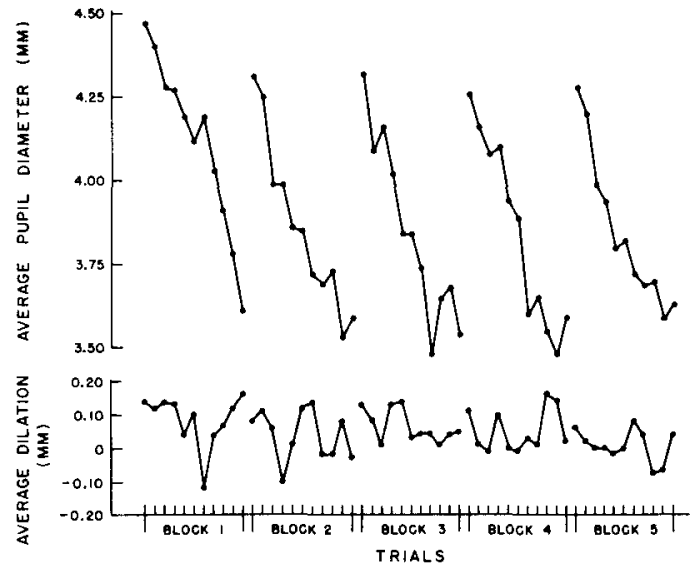

Fig. 3. Top curve: average pupillary diameter at the instant of presentation of the ready signal, for successive experimental trials. Bottom curve: average dilation for one second following ready signal.

the light appears sufficient to restore pupil size to a reasonably constant baseline.

The occurrence of important baseline shifts within each block of trials raises the possibility of serious artifacts: is the significance of a particular dilation affected by a change of baseline? The lower graph in Fig. 3 does not support such a conclusion; statistical tests indicate that the within-block trend, which is hugely significant for the baseline level $(F=227.47$, $\mathrm{df}=1 / 90$ ), does not approach significance for either the dilation to the ready signal $(F=1.50)$ or the dilation to the standard tone $(F=0.18)$. The conclusion appears justified that within-block constriction does not present a severe problem in the interpretation of pupillary dilations, although it is a factor that must be controlled in the design of pupillary investigations.

While the preceding discussion of baseline changes has been motivated by a concern with possible artifacts, the occurrence of changes in the magnitude of pupillary dilations presents a psychologically significant issue. As was noted above, the design of the present experiment was such that the standard tone became gradually less informative with repeated trials. Consequently, the responses to this tone and to the ready signal which always preceded it were expected to diminish gradually during the session. On the other hand, subjective reports indicated that the discriminations did not become substantially easier with practice, although the number of errors decreased significantly between the first and the last block $(F=6.87$, df $=1 / 36, \quad p<.01)$. We therefore expected the response to the comparison tone to remain essentially constant. Figure 4 shows that these expectations were confirmed. A decreasing linear trend is significant for the magnitude of the response to the ready signal $(F=4.72, \mathrm{df}=1 / 36, \mathrm{p}<.05)$ and for the response to the standard tone $(F=13.92, p<.001)$ but such a 


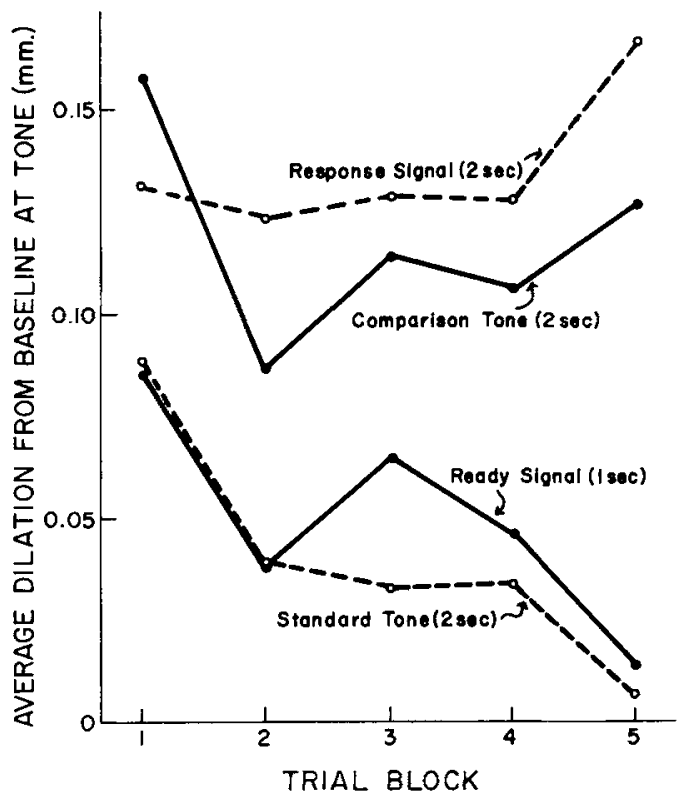

Fig. 4. Average dilations to the different stimuli as a function of trial block. The number of seconds over which dilation is measured is indicated for each curve.

trend is not found for the dilation to either the comparison tone $(F=0.45, N S)$ or the instruction to respond ( $F=2.85, N S)$. Evidently, a reduction of the pupillary response is a correlate of reduced psychological significance of the stimulus, and is not an effect of mere repetition. Similar conclusions have been reached (Walter, 1964; Cohen \& Walter, 1966) in the description of the evoked potentials that accompany psychologically significant events.

\section{Discussion}

Until recently, pupillary dilations have been discussed mainly in the context of descriptions of emotional arousal (Hess \& Polt, 1960, 1966; Hess, Seltzer, \& Shlien, 1965) or the orienting response (Sokolov, 1963). The present research adds to the growing evidence that a third important class of pupillary dilations must be considered, which are closely related to the demands that mental tasks of various kinds place on the information-processing capacity of the organism. The magnitude of the responses that have been described here is clearly not determined by the arousing characteristics of any stimulus; rather, it corresponds to what the organism does with the information conveyed by a particular stimulus. The pupillary responses with which we are concerned are probably linked to the EEG correlates of specific mental activity which have recently been described (Davis, 1964; Haider, Spong, \& Lindsley, 1964; Sutton, Zubin, \& John, 1965; Walter, 1964).

The frequent use of the concept of processing load in the present paper has been guided by a simple analogy: consider a houseful of electrical devices that are variously put in operation by manual switches or by their own internal governor systems. The total amperage demanded by the entire system at any one time may easily be read on an appropriate electrical instrument outside the house. Processing load is here construed as analogous to an aggregate demand for power, and there is ground for the hope that the pupil may function as a useful approximation to the relevant measuring device. There is an important difference between the contexts in which the concept of load and the more familiar concept of arousal would be applied. An organism may be said to be aroused by a stimulus; an organism is placed under load by its own responses.

In several places in this paper, alternative interpretations of a specific pupillary response have been mentioned, either in terms of processing load or in terms of an emotional response. One may even attempt to extend the emotional interpretation to all pupillary changes, claiming that they reflect changing levels of the anxiety that accompanies performance. This interpretation is hard to disprove, since it is probably true that the performance of more difficult tasks is generally accompanied by a higher level of anxiety. However, the proponents of such a view may have to broaden the concept of anxiety so much as to make it either virtually meaningless or else a synonym of processing load. In particular, it is certainly the case that some people find the challenge of dealing with mental problems altogether attractive and pleasant, and yet respond to these activities by major pupillary dilations. Our current impression is that pupillary dilations probably accompany any substantial increase of mental activity, regardless of the pleasantness or unpleasantness of this activity.

\section{References}

Beatty, J., \& Kahneman, D. Pupillary changes in two memory tasks. Psychon. Sci., 1966, 5, 371-372.

Cohen, J., \& Walter, W. G. The interaction of responses in the brain to semantic stimuli. Psychophysiology, 1966, 2, 187-196.

Davis, H. Enhancement of evoked cortical potentials in humans related to a task requiring a decision. Science, 1964, 144, 182183.

Haider, M., Spong, P., \& Lindsley, D. B. Attention, vigilance, and cortical evoked-potentials in humans. Science, 1964, 144, 180182.

Hess, E. H., \& Polt, J. M. Pupil size as related to interest value of visual stimuli. Science, 1960, 132, 349-350.

Hess, E. H., \& Polt, J. M. Pupil size in relation to mental activity during simple problem-solving. Science, 1964, 143, 1190-1192.

Hess, E. H., \& Polt, J. M. Changes in pupil size as a measure of taste difference. Percept. mot. Skills, 1966, 23, 451-455.

Hess, E. H., Seltzer, A. L., \& Shlien, J. M. Pupil response of hetero- and homosexual males to pictures of men and women: A pilot study. J. abnorm. Psychol., 1965, 70, 165-168.

Kahneman, D., \& Beatty, J. Pupil diameter and load on memory. Science, 1966, 154, 1583-1585.

Lacey, J. I., Kagan, J., Lacey, B. C., \& Moss, H. A. The visceral level: Situational determinants and behavioral correlates of 
auto nomic response patterns. In P. H, Knapp (Ed.), Expression of the emotions in man. New York: International Universities Press, 1963.

Obrist, P. A. Cardiovascular differentiation of sensory stimuli. Psychosom. Med., 1963, 6, 450-459.

Paivio, A., \& Simpson, H. M. The effect of word abstractness and pleasantness on pupil size during an imagery test. Psychon. sci., 1966, 5, 55-56.

Sokolov, E. N. Higher nervous functions: the orienting reflex. Ann. Rev. Physiol., 1963, 25, 545-580.

Stevens, S. S. Problems and methods of psychophysics. Psychol. Bull, , 1958, 54, 177-196.

Sutton, S., Braren, M., Zubin, J., \& John, E. R. Evoked-potential correlates of stimulus uncertainty. Science, 1965, 150, 1187-1188.

Walter, W. G. Slow potential waves in the human brain associated with expectancy, attention and decision. Arch. Psychiat. Z. ges Neurol., 1964, 206, 309-322.

Wickelgren, W. A. Consolidation and retroactive interference in short-term recognition memory for pitch. J. exp. Psychal., 1966, $72,250-259$.

\section{Note}

1. This research was supported in part by the Advanced Research Projects Agency, Department of Defense, and was monitored by the Air Force Office of Scientific Research, under Contract \#AF 49 (638) 1235 with the Human Performance Center, Department of Psychology, University of Michigan. Analysis of the data was supported in part by a contract with the United States Department of Health, Education and Welfare, Office of Education, under the provisions of the Cooperative Research Program, Contract \# 6-10-043, Project Z-014, and by a grant from the National Institute of Mental Health, Grant \#P01-MH-12623, to Harvard, University, Center for Cognitive Studies.

(Accepted for publication December 13, 1966.) 